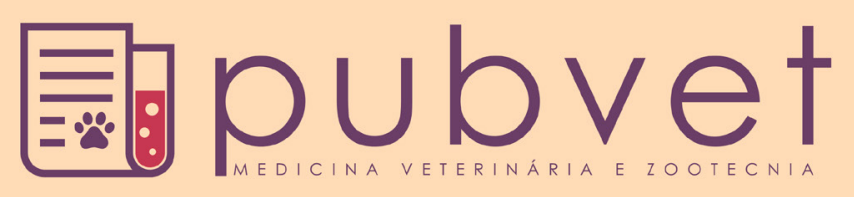

HTTP://DX.DOI.ORG/10.22256/PUBVET.V11N6.596-600

\title{
Histerocele gravídica diafragmática em felino: Relato de caso
}

\author{
Marina de Carvalho Cavalcanti ${ }^{1}$, Larissa Sasaki Yamaguchi ${ }^{2}$, Ângela Maria da Silva ${ }^{3}$, \\ Alexandre Coltro Gazzone ${ }^{4}$, Desireé Reis de Oliveira ${ }^{2}$, Paulo Antonio Terrabuio \\ Andreussi $^{*}$
}

Universidade Federal de Mato Grosso do Sul, Faculdade de Medicina Veterinária e Zootecnia, Campo Grande, MS, Brasil.

${ }^{1}$ Médica Veterinária Residente em Ginecologia e Obstetrícia Veterinária. ${ }^{2}$ Médica Veterinária residente em Diagnóstico por Imagem. ${ }^{3}$ Médica Veterinária Residente em Anestesiologia e Emergência. ${ }^{4}$ Graduando em Medicina Veterinária. ${ }^{5}$ Docente de Diagnóstico por Imagem e Obstetrícia Veterinária.*Autor para correspondência: pauloandreussi@hotmail.com

RESUMO. Descreve-se um caso de atendimento a animal, felino, adulto, fêmea, com diagnóstico de hérnia diafragmática crônica tendo o útero gravídico em fase final de gestação como parte do conteúdo herniário. O gradativo crescimento fetal no decorrer da gestação determinou importante comprometimento respiratório. A gravidade do quadro exigiu interrupção cirúrgica da gestação com posterior herniorrafia por flape peritoneal, porém não houve êxito e o animal veio a óbito ao final do procedimento cirúrgico. $\mathrm{O}$ óbito foi atribuído à cronicidade e gravidade da condição clínica em que se encontrava. Este relato ressalta a importância da correção imediata de hérnias diafragmáticas e do acompanhamento pré-natal durante a gestação.

Palavras chave: Dispneia, interrupção gestacional, trauma torácico

\section{Diaphragmatic hernia containing pregnant uterus as content in feline: Case report}

ABSTRACT. A case of animal care, feline, adult, female is described, with diagnosis of chronic diaphragmatic hernia with the gravid uterus in the final stage of gestation as part of the hernial content. Gradual fetal growth during pregnancy led to significant respiratory impairment. The severity of the condition necessitated a surgical interruption of gestation with posterior herniorrhaphy with peritoneal flap, but it did not succeed and the animal died at the end of the surgical procedure. The death was attributed to the chronicity and severity of the clinical condition in which it was found. This report highlights the importance of immediate correction of diaphragmatic hernias and prenatal follow-up during pregnancy.

Keyword: Dyspnea, gestational interruption, thoracic trauma

\section{Histerocele diafragmática en una gata gestante: Reporte de un caso}

RESUMEN. Se describe el reporte de un caso de una felina adulta, en fase final de gestación con diagnóstico de hernia diafragmática crónica conteniendo útero con fetos. El progresivo crecimiento fetal durante el transcurso de la gestación determinó una importante complicación respiratoria. La gravedad del cuadro requirió de la interrupción quirúrgica de la gestación con posterior herniorrafia por flape peritoneal, pero no hubo éxito y el animal murió al final del procedimiento quirúrgico. El fallecimiento fue atribuido a la cronicidad y gravedad de la condición clínica en que se encontraba. Este reporte resalta la importancia de la corrección inmediata de hernias diafragmáticas y del acompañamiento prenatal durante la gestación.

Palabras clave: Disnea, interrupción gestacional, trauma torácico 


\section{Introdução}

A hérnia diafragmática ocorre quando o diafragma é rompido, resultando na migração dos órgãos abdominais para o interior da cavidade torácica. As hérnias diafragmáticas podem ser de origem congênita ou secundariamente ao trauma (Fossum, 2014). Quando o conteúdo herniário é o útero, isoladamente ou em associação com outras estruturas, configura-se em histerocele.

O contato do espaço pleural com os pulmões normalmente é mantido por pressão negativa, porém, com o rompimento do diafragma esta pressão é perdida, fazendo com que os músculos abdominais e torácicos assumam a função do diafragma, e as pressões peritoneal e pleural se igualem (Mushin, 1980), resultando assim, em dificuldade respiratória significativa. Entretanto, animais assintomáticos com hérnias diafragmáticas crônicas não são incomuns (Fossum, 2014). A extensão da ruptura e a quantidade de vísceras abdominais presentes no tórax estão interligadas com os sintomas apresentados pelo animal, sendo a dispneia o principal distúrbio e o mais descrito em literatura. Outros sinais também são encontrados, como inquietação, estação com relutância em deitar ou andar (Bojrab, 2005).

A hérnia diafragmática pode permanecer não diagnosticada caso os sinais sejam tão discretos que fujam à percepção do proprietário, levando a situações mais complexas nos casos de prenhez ou em procedimentos anestésicos em animais não diagnosticados como portadores de hérnia diafragmática crônica (Ricco and Graham, 2007).

O fígado é o órgão mais frequentemente herniado, sendo encontrado na cavidade torácica em 81 a $88 \%$ dos pacientes. Outros órgãos comumente encontrados em ordem decrescente são o intestino delgado (67\%), estômago (48\%), omento $(38,5 \%)$, baço $(25 \%)$, pâncreas $(13,5 \%)$, intestino grosso (8\%) (Besalti et al., 2011) e útero (Slatter, 2007). Embora o diagnóstico seja baseado no histórico, com possíveis relatos de trauma (Fossum, 2014), e em sinais clínicos, o exame radiográfico é indispensável para a confirmação do diagnóstico clínico (Kealy et al., 2005). O tratamento consiste primeiramente na estabilização clínica do animal caso se encontre desidratado e / ou dispneico, sendo assim, deverá receber oxigênio e fluidoterapia e, somente depois ser submetido ao procedimento cirúrgico de reconstituição do diafragma (Fossum, 2014).
A síntese por aproximação das bordas da ferida é suficiente para a cicatrização do músculo, porém, defeitos com maior extensão de lesão, contração muscular e retração cicatricial não permitem a aproximação das bordas para a síntese, como por exemplo, em casos de necrose decorrente de infecção, traumatismo, extensa ressecção do músculo por neoplasia e nas hérnias crônicas, sendo indicada a hernioplastia (Barreiros et al., 1996). Após o procedimento cirúrgico a reexpansão pulmonar rápida pode provocar edema pulmonar, devendo-se considerar o evento como uma complicação em potencial (Fossum, 2014). A taxa de mortalidade associada à hérnia diafragmática é variável e tem sido reduzida em comparação com relatos de publicações mais antigas. Estudos apontam taxa de mortalidade, em gatos, de aproximadamente 16 a $20 \%$ em casos agudos e 11,8 a $19 \%$ em crônicos (Downs and Bjorling, 1987, Besalti et al., 2011, Legallet et al., 2017). Aparentemente, o tempo decorrido até a cirurgia não interfere na taxa de sobrevivência, mas aqueles animais com maior volume de órgãos herniados para a cavidade torácica e maior grau de lesões em tecidos moles tiveram menor taxa de sobrevida em todo o período perioperatório (Besalti et al., 2011). Contrariamente, Fossum (2014) não recomenda o adiamento da correção cirúrgica, alegando maior taxa de mortalidade em casos crônicos.

Este relato tem como objetivo apresentar um caso de histerocele gravídica diafragmática em felino, tendo em vista a escassez de relatos anteriores.

\section{Casuística}

Foi atendido no setor de Ginecologia e Obstetrícia do Hospital Veterinário da Universidade Federal de Mato Grosso do Sul, um felino, fêmea, sem raça definida, dois anos de idade, com histórico de acasalamento há quase dois meses, inapetência; e trauma por acidente automobilístico há um ano, resultando em hérnia diafragmática. Segundo a proprietária, à época, a hérnia foi diagnosticada, porém, em virtude da melhora do quadro clínico do animal e questões financeiras, optou-se pela não realização do procedimento cirúrgico. Ao exame físico o animal apresentou dispneia, ausculta pulmonar abafada, caquexia e leve desidratação. Como exame complementar foi solicitado exame radiográfico de tórax, no qual foi confirmada a presença de hérnia diafragmática evidenciando o útero gravídico como componente do conteúdo (Fig. 1). 


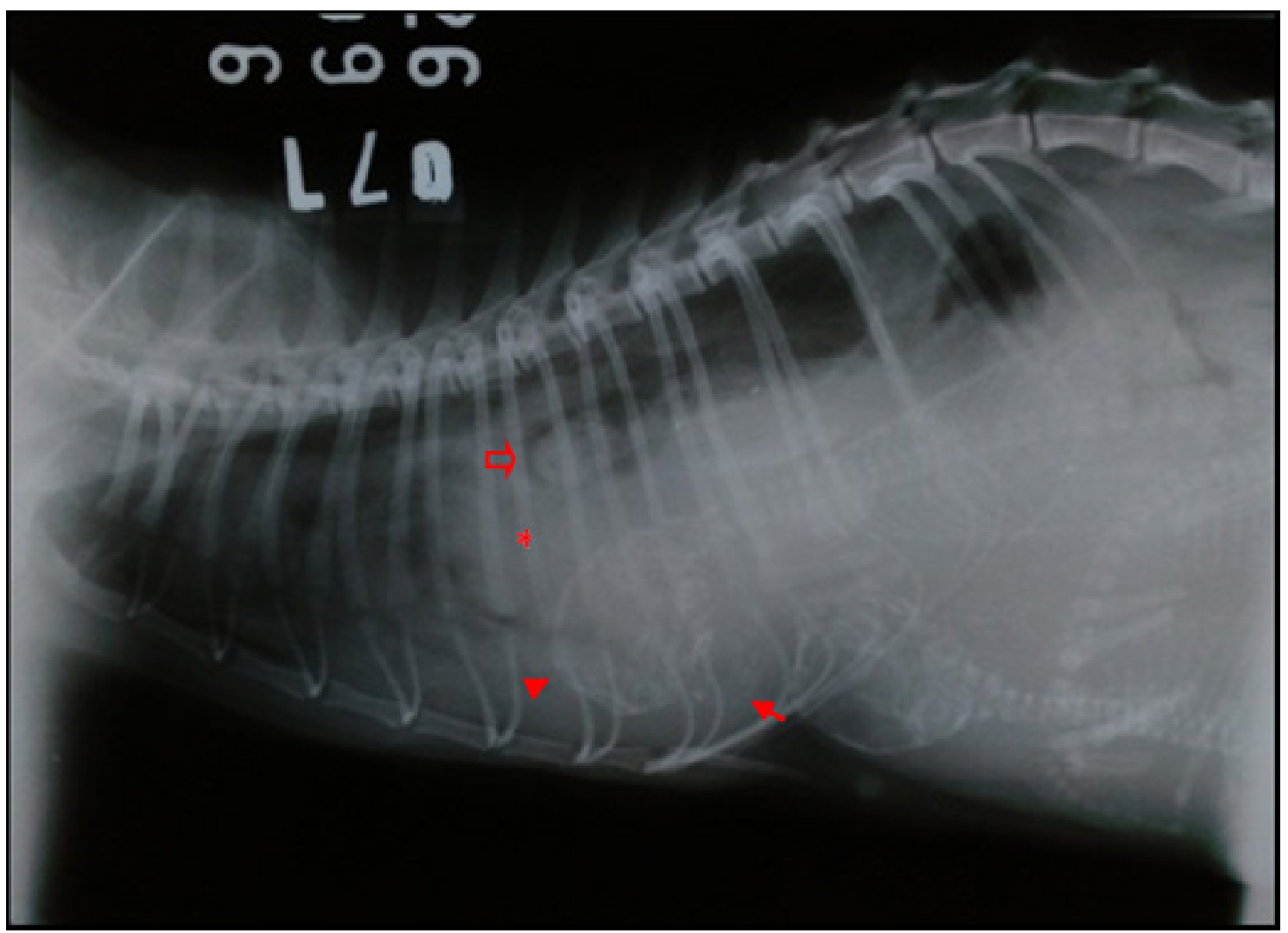

Figura 1. Radiografia torácica em projeção laterolateral direita evidenciando radiopacidade no aspecto caudoventral do tórax, com perda de definição de cúpula. Nota-se presença de estruturas fetais (crânio já mineralizado - ponta de seta), segmento intestinal (seta larga), região com opacidade água correspondendo a liquido fetal (seta fina). A silhueta cardíaca deslocada dorsalmente (asterisco)

Devido à gravidade do quadro, com extenso preenchimento de campos pulmonares (Figura 1) e dispneia grave, a intervenção necessária foi a interrupção cirúrgica da gestação, e posterior herniorrafia. O procedimento foi realizado por laparotomia mediana supra-umbilical sob ventilação mecânica. Durante a exteriorização do útero notou-se que não havia aderência deste às estruturas diafragmáticas, e o mesmo foi deslizado livremente. O diafragma apresentava-se atrofiado, com grande retração das bordas, necessitando realização de hernioplastia por enxerto (flape abdominal). $\mathrm{O}$ animal veio a óbito durante a finalização dos procedimentos, após a síntese da parede abdominal, provavelmente por complicações decorrentes da reexpansão pulmonar.

\section{Discussão}

A hérnia diafragmática aguda pode determinar quadros graves, com comprometimento cardiorrespiratório importante e choque, porém, alguns animais podem apresentar quadros, inicialmente, menos intensos e permanecerem cronicamente com a lesão, apresentando apenas quadros clínicos mais discretos, tais como dispneia leve, intolerância ao exercício, dificuldade em se alimentar quando estruturas gástricas são herniadas e emagrecimento (Fossum, 2014). No animal em questão não houve percepção da maioria destes sinais pelo proprietário, exceto emagrecimento, com isso, o risco que a atividade sexual poderia trazer foi negligenciado, inclusive após a ocorrência do acasalamento.

A ocorrência de gestação em animais portadores de hérnia diafragmática crônica pode agravar sobremaneira sua condição clínica, principalmente pelo crescente aumento da pressão intra-abdominal decorrente do desenvolvimento fetal. O crescimento fetal em felinos, embora seja considerado linear, é expressivamente maior no terço final da gestação, de forma que menos de $30 \%$ do crescimento do seu comprimento ocorre durante as primeiras cinco ou seis semanas de gestação (Evans et al., 1998).

A mineralização esquelética fetal observada na imagem radiológica (Figura 1). A imagem que neste caso o animal se encontrava no terço final da gestação e independentemente de ter havido algum comprometimento orgânico deste foi o 
estado gestacional quem desencadeou o agravamento clínico, obrigando a conduta terapêutica radical que resultou em óbito. Esta hipótese que pode ser justificada pelo grande volume de vísceras dentro do tórax, inclusive útero, o que pode agravar o prognóstico, segundo Besalti et al. (2011).

A cronicidade da hérnia diafragmática não é apontada como um fator de agravamento do prognóstico por alguns autores (Downs and Bjorling, 1987, Besalti et al., 2011); porém, segundo Fossum (2014) estes casos podem ter taxa de mortalidade mais alta do que as hérnias diafragmáticas agudas, não sendo recomendado o adiamento da correção cirúrgica sem um motivo relevante.

Após a segunda semana da ruptura, o tecido diafragmático sofre formações de aderências com elevado nível de tecido fibrovascular organizado (Zimmermann et al., 2008), impossibilitando a aproximação das bordas durante a herniorrafia simples, com aumento da tensão na linha de sutura e inevitável predisposição à deiscência dos planos de sutura, dificultando a cicatrização por primeira intenção. Nestes casos, são necessários procedimentos cirúrgicos mais apropriados, como a utilização de enxertos (Mazzanti et al., 2001) o que coincide com o observado no animal atendido. Os procedimentos mais prolongados, com maior duração do período de anestesia também estão relacionados com o agravamento do prognóstico (Besalti et al., 2011).

\section{Considerações finais}

Neste relato fica clara a importância da correção cirúrgica das hérnias diafragmáticas no mais breve prazo possível, mesmo que não haja sinais clínicos importantes, e também a gravidade dos efeitos que a gestação provoca em fêmeas portadoras desta condição na forma crônica. O diagnóstico da gestação precoce e exames prénatais são fundamentais para nortear a condução de diversas situações na prática médica e cirúrgica de pequenos animais, como em casos tais como o aqui relatado. Caso a gestação tivesse sido diagnosticada precocemente, os procedimentos terapêuticos poderiam ser executados com a paciente em condições mais favoráveis, e o êxito seria diferente do apresentado. Os exames por imagem são fundamentais em exames pré-natais em pequenos animais.

\section{Referências Bibliográficas}

Barreiros, L. J., Rodaski, S., Susko, I., Werner, P. R. \& Messias, C. 1996. Uso experimental do músculo grande dorsal autólogo na reparação dos grandes defeitos diafragmáticos no cão. Revista do Setor de Ciências Agrárias, 15, 141-150.

Besalti, O., Pekcan, Z., Caliskan, M. \& Aykut, Z. G. 2011. A retrospective study on traumatic diaphragmatic hernias in cats. Ankara Univ Vet Fak Derg, 58, 175-179.

Bojrab, M. J. 2005. Técnicas atuais em cirurgia de pequenos animais. Editora Roca, São Paulo.

Downs, M. \& Bjorling, D. 1987. Traumatic diaphragmatic - hernias - a review of 1674 casos. Veterinary Surgery. WB Saunders, Philadelphia.

Evans, S. D., Nott, K. P., Kshirsagar, A. A. \& Hall, L. D. 1998. The effect of freezing and thawing on the magnetic resonance imaging parameters of water in beef, lamb and pork meat. International Journal of Food Science \& Technology, 33, 317-328.

Fossum, T. W. 2014. Cirurgia de pequenos animais, 4 edn. Elsevier Brasil, São Paulo.

Kealy, J. K., McAllister, H. \& Graham, J. P. 2005. Radiologia e Ultrassonografia do Cão e do Gato. Manole, São Paulo.

Legallet, C., Mankin, K. T. \& Selmic, L. E. 2017. Prognostic indicators for perioperative survival after diaphragmatic herniorrhaphy in cats and dogs: 96 cases (2001-2013). BMC Veterinary Research, 13, 16.

Mazzanti, A., Pippi, N. L., Raiser, A. G., Graças, A. F. S., Faria, R. X., Alves, A. S., Gonçalves, G. F., Selides, R. \& Bragas, F. A. 2001. Músculo diafragma homólogo conservado em solução supersaturada de açúcar para reparação de grande defeito no diafragma de cão. Ciência Rural, 31, 277-283.

Mushin, W. W. 1980. Automatic ventilation of the lungs. Blackwell Scientific, Oxford.

Ricco, C. H. \& Graham, L. 2007. Undiagnosed diaphragmatic hernia - the importance of preanesthetic evaluation. The Canadian Veterinary Journal, 48, 615-618.

Slatter, D. H. 2007. Manual de cirurgia de pequenos animais. Manole, São Paulo. 
Zimmermann, M., Raiser, A. G., Braga, F. V., Trindade, A. L. \& Lopes, S. T. 2008. Membranas de látex natural na herniorrafia diafragmática experimental em cães. Arquivo Brasileiro de Medicina Veterinaria $e$ Zootecnia, 60, 1476-1483.
Article History:

Received 23 February 2017

Accepted 3 April 2017

Available on line 29 May 2017

License information: This is an open-access article distributed under the terms of the Creative Commons Attribution License 4.0, which permits unrestricted use, distribution, and reproduction in any medium, provided the original work is properly cited. 